\title{
Statistical analysis and data mining of digital reconstructions of dendritic morphologies
}

\author{
Sridevi Polavaram, Todd A. Gillette, Ruchi Parekh and Giorgio A. Ascoli *
}

Department of Molecular Neuroscience, Center for Neural Informatics, Structures, and Plasticity, Krasnow Institute for Advanced Study, George Mason University, Fairfax, VA, USA

\section{Edited by:}

Hermann Cuntz, Ernst Strüngmann Institute in Cooperation with Max Planck Society, Germany

Reviewed by:

Corinne Teeter, Allen Institute for Brain Science, USA

Marcel Oberlaender, Max Planck Institute for Biological Cybernetics, Germany

*Correspondence:

Giorgio A. Ascoli, Molecular Neuroscience Department, Center for Neural Informatics, Structures, and Plasticity, Krasnow Institute for Advanced Study, George Mason University, MSN 2A1, 4400 University Dr. Fairfax, VA 22030 USA

e-mail:ascoli@gmu.edu
Neuronal morphology is diverse among animal species, developmental stages, brain regions, and cell types. The geometry of individual neurons also varies substantially even within the same cell class. Moreover, specific histological, imaging, and reconstruction methodologies can differentially affect morphometric measures. The quantitative characterization of neuronal arbors is necessary for in-depth understanding of the structure-function relationship in nervous systems. The large collection of community-contributed digitally reconstructed neurons available at NeuroMorpho.Org constitutes a "big data" research opportunity for neuroscience discovery beyond the approaches typically pursued in single laboratories. To illustrate these potential and related challenges, we present a database-wide statistical analysis of dendritic arbors enabling the quantification of major morphological similarities and differences across broadly adopted metadata categories. Furthermore, we adopt a complementary unsupervised approach based on clustering and dimensionality reduction to identify the main morphological parameters leading to the most statistically informative structural classification. We find that specific combinations of measures related to branching density, overall size, tortuosity, bifurcation angles, arbor flatness, and topological asymmetry can capture anatomically and functionally relevant features of dendritic trees. The reported results only represent a small fraction of the relationships available for data exploration and hypothesis testing enabled by sharing of digital morphological reconstructions.

Keywords: L-Measure (RRID:nif-0000-00003), NeuroMorpho.Org (RRID:nif-0000-00006), neuroinformatics, dendritic topology, cluster analysis, cellular neuroanatomy

\section{INTRODUCTION}

The diversity of neuronal morphologies can have broad and profound functional consequences in the nervous system, which have only begun to be understood. Dendritic geometry directly impacts (and mediates) computational processes such as signal integration, coincidence detection, and logical operations (London and Häusser, 2005). The location, orientation, and shape of neural arbors enable (and strongly affect) network connectivity, providing the anatomical substrate to investigate structure-function relationship at the circuitry level (Shepherd and Svoboda, 2005; Briggman and Denk, 2006; Kajiwara et al., 2008; Weiler et al., 2008; Burgalossi et al., 2011; Ropireddy and Ascoli, 2011; Brown et al., 2012). These areas of scientific investigation apply to the morphological differences observed both within and between neuron types across animal species, developmental stages, and brain regions (Figure 1).

Three-dimensional digital reconstructions of axonal and dendritic arbors, combined with neuroinformatics tools and computational approaches, allow considerable opportunities for data processing, analysis, and modeling at both cellular- and systems-level (Parekh and Ascoli, 2013). The open availability of these reconstructions in databases such as NeuroMorpho.Org (Figure 2) enables re-analysis of shared data (Ascoli, 2007). As of version 5.6, the repository contained over 10,000 reconstructions contributed by 120 laboratories from 21 species, 85 brain regions and 123 cell types, representing more than 240,000 hours of manual tracing. NeuroMorpho.Org users can browse the data by animal species, brain region, cell type, and contributing lab. The "search by" option can be used to select and combine specific metadata criteria (Figure 2, left panel top) from a drop-down menu of categories such as developmental stage, experimental condition, and reconstruction method. The morphometry search functionality (Figure 2, left panel bottom) allows users to find neurons matching any combination of more than 20 morphometric criteria. From the resulting summary list of neurons (Figure 2, middle panel), individual pages for each reconstruction can be retrieved, thus displaying related metadata, a link to the associated publication, and the pre-computed morphometrics (Figure 2, right panel). Each reconstruction is downloadable as the standardized version along with the original contributed version. The log files detailing the changes made during the standardization process are available for download as well. From the individual neuron pages, users can also launch an animation file and an interactive $3 \mathrm{D}$ viewer.

Quantitative morphometry of neuronal reconstructions is often used for shape analysis (Uylings and van Pelt, 2002; 


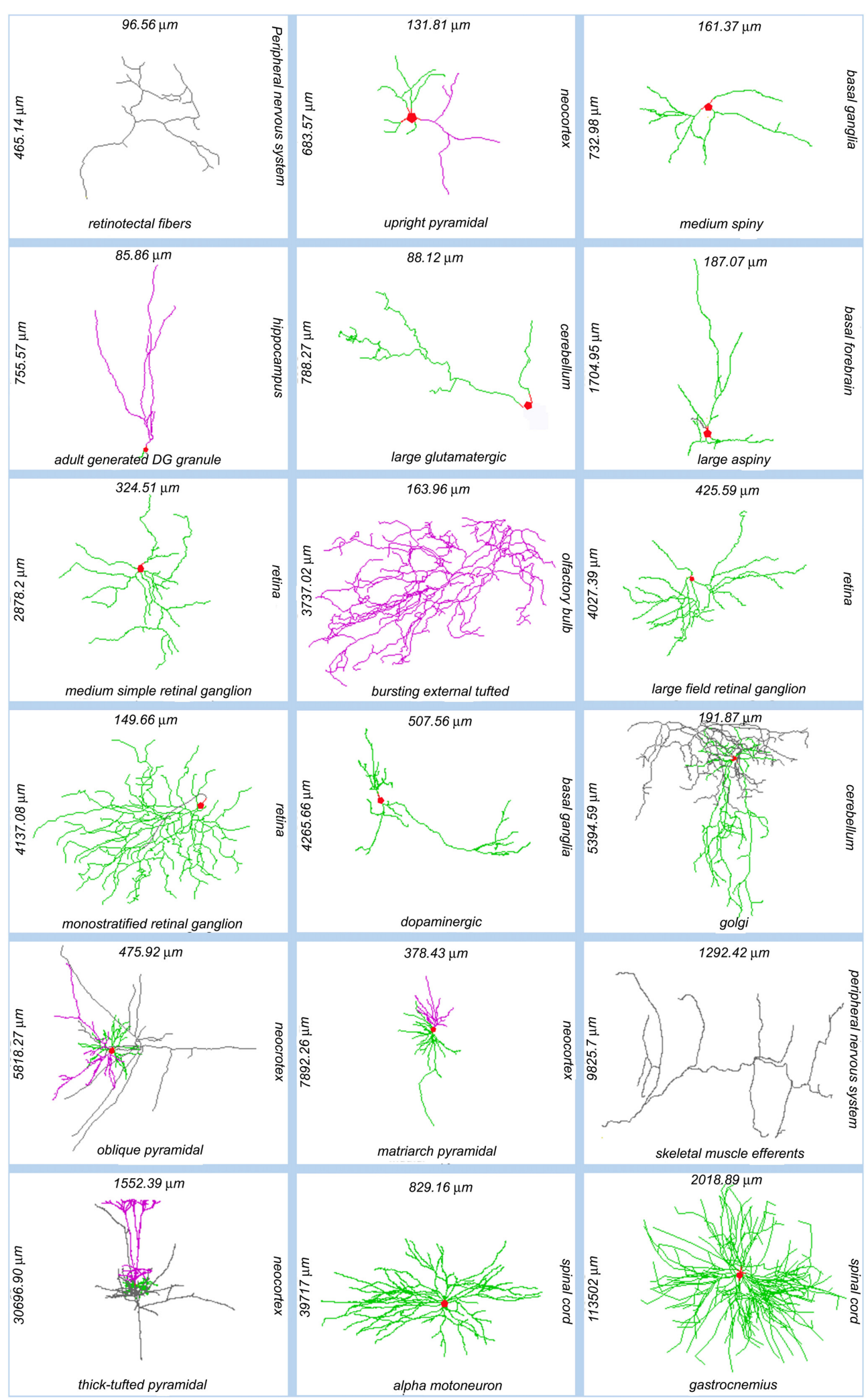

FIGURE 1 | Sample of NeuroMorpho.Org reconstructions representing the anatomical diversity of dendritic and axonal trees. Each image is labeled (clockwise from its right side) with the somatic brain region, neuron types, total arbor length, and arbor width. Somata: red; axons: gray; (basal) dendrites: green; apical dendrites: magenta. NeuroMorpho.Org IDs of these neurons from left to right: 06787, 04183, 04457, 06312,

05713, 04477, 00779, 06216, 00777, 05491, 00888, 06904, 06141, 06295, 07707, 07763, 00690, 00606 


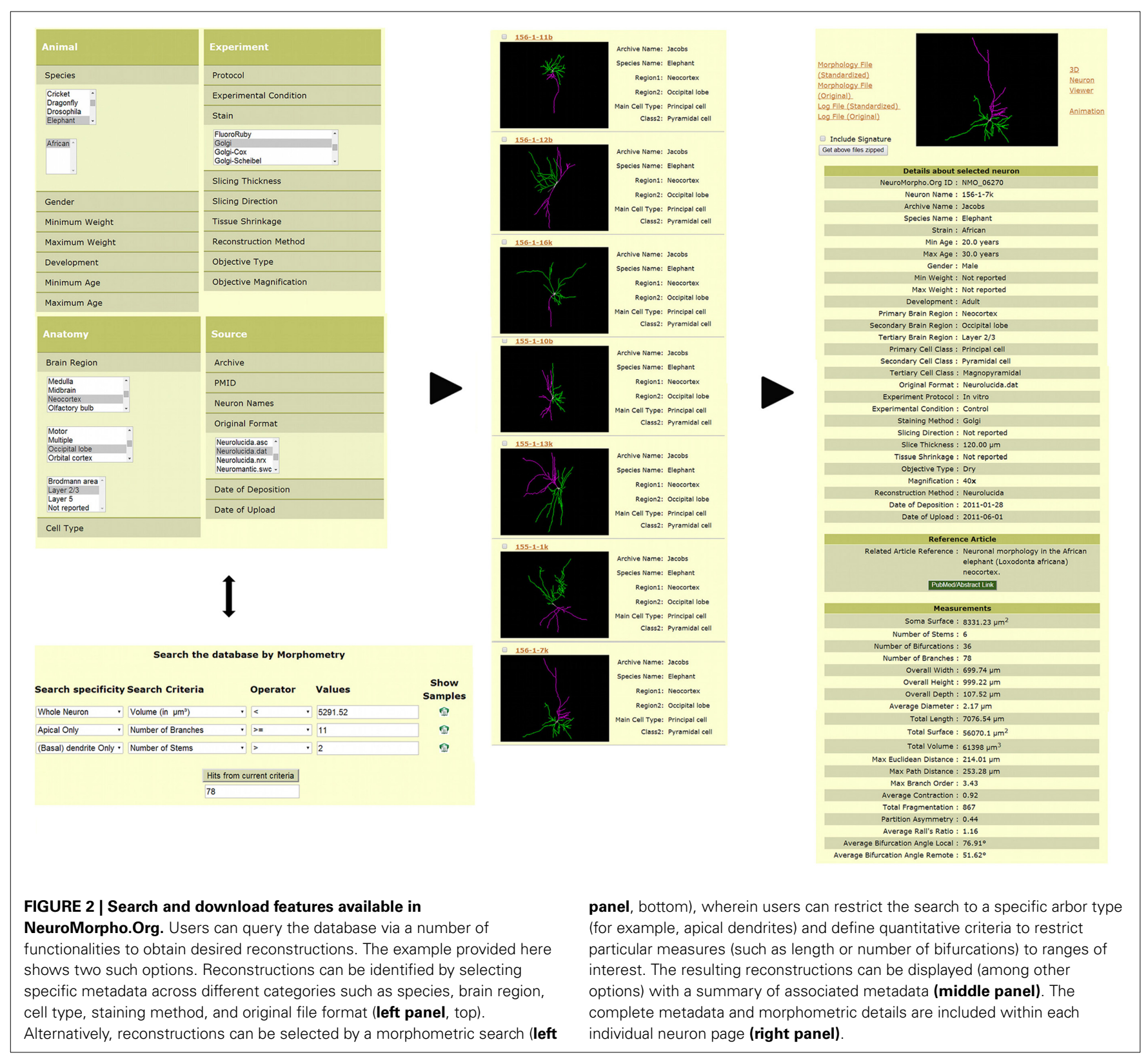

Van Ooyen et al., 2002; Rocchi et al., 2007), also in conjunction with biologically-inspired computational simulations (Ascoli et al., 2001; Van Ooyen, 2011). For example, statistical distribution of morphological features are used in stochastic growth algorithms for generating virtual trees (Van Pelt et al., 1997; Donohue and Ascoli, 2008; Koene et al., 2009; Evans and Polavaram, 2013; Memelli et al., 2013). Moreover, statistical analyses of neuronal reconstructions facilitate and support theoretical investigations. These studies for instance provided evidence for optimal wiring principles of neuronal arbors (Wen and Chklovskii, 2008) and their power law distributions, which may relate to synaptic input sampling (Lee and Stevens, 2007; Snider et al., 2010; Teeter and Stevens, 2011; Cuntz et al., 2012).

This study uses the L-Measure software tool (Scorcioni et al., 2008) to extract morphometric data from neuronal arbors for large scale statistical analyses of available data. L-Measure computes simple statistics of morphometric features as well as their frequency distribution and inter-dependence (e.g., how arbor length varies with path distance from the soma). This tool has been used in a broad range of applications, including multidimensional analysis of neuronal shape (Costa et al., 2010; Zawadzki et al., 2012) and comparative studies of sensory neurons in the fly (Ting et al., 2014) and of respiratory neurons in the pre-Bötzinger complex (Koizumi et al., 2013). In conjunction with L-Neuron (Ascoli and Krichmar, 2000), L-Measure has also been employed to generate and validate a large-scale model of the dentate gyrus with half a million neurons (Schneider et al., 2012). L-Measure has also enabled analysis of non-neuronal arbors such as arterial vasculature (Wright et al., 2013), and was integrated into other digital reconstruction and analysis systems, such as the 
Farsight toolkit (http://farsight-toolkit.org) and Vaa3D (https:// code.google.com/p/vaa3d).

With the first successes in high-throughput automatic digital neuronal tracing (Chiang et al., 2011) and overall increasing volumes of published and shared reconstructions (Halavi et al., 2012), "big data" opportunities for knowledge mining are starting to emerge. On the one hand, this increasing availability of shared data may foster remarkable discoveries. On the other, the heterogeneous source of data and disparate experimental conditions also pose non-trivial challenges to database-wide analyses. As a step toward large database analysis, here we utilize exploratory data analysis to quantify morphological similarities and differences across broadly diverse dendritic arbors. In the process, we recognize several critical limitations when pooling together widely non-uniform data sets. Consequently, we propose selection criteria and methodological choices aimed to maximize the potential biological relevance of the results. With such a research design, dimensionality reduction and unsupervised clustering reveal tentative morphological relationships between specific neuron types involving branching density, topology, size, and tortuosity. At the same time, we identify the most delicate factors in both data and metadata that must be considered to optimize the impact of future large-scale morphological investigations.

\section{METHODS \\ SELECTION OF DATASETS AND MORPHOMETRIC FEATURES FOR ANALYSIS}

The entire pool of 10,004 reconstructions downloaded from NeuroMorpho.Org v5.6 was screened for a pre-determined set of inclusion criteria to improve interpretability of the results. Specifically, in order to be considered for analysis, digital neuron reconstructions had to (a) belong to the "control" experimental condition; (b) contain at least four dendritic bifurcations; (c) include branch-path information and not just bifurcation connectivity; and (d) have non-zero depth range (eliminating two-dimensional tracings). The 7,143 reconstructions matching these characteristics were analyzed by their NeuroMorpho.Org metadata assignments to specific animal species, brain region, and cell type. Subsequently, for the purpose of cluster analysis chi-square testing (see below), groups of fewer than 40 neurons in any metadata combination of species, brain region, cell type, and lab of origin were excluded to ensure sufficient statistical power (Yates et al., 1999). This further selection reduced the number of reconstructions to 5,099 , divided into 45 unique metadata groups.

Because of the major differences between axonal and dendritic morphology, and the remarkable abundance of reconstructed dendrites relative to axons, only dendritic arbors were included in this study. Focusing on a more consistent and comparable dataset allows addressing more biologically relevant questions. Moreover, this choice reduces the errors due to incomplete reconstructions, which are considerably more severe for projection axons than for dendrites.

L-Measure allows extraction of approximately 100 distinct features from each neuron (see http://cng.gmu.edu:8080/Lm for full listing and detailed definitions). Of these, all measures related to branch diameter were excluded due to their strong dependence on imaging resolution, optical magnification, and other experimental details causing excessive inter-laboratory variability (Scorcioni et al., 2004). All other features were subjected to cross-correlation analysis, and those with correlation greater than $80 \%$ were sequentially eliminated one at a time (re-running the cross-correlation at each step) as they were considered highly redundant with the rest of the features. This selection left 27 features (Table 1) that were used for the remainder of the analysis. Dendritic arbor size measures consisted of total length, number of tips, height, width, and depth. Bifurcation measures included average partition asymmetry as well as amplitude, tilt, and torque angles measured locally with the adjacent tracing points or remotely with the preceding and following bifurcations or terminations. Branch measures consisted of length, tortuosity, and fractal dimension. Lastly, local measures included branch order, terminal degree, path distance from soma, and helicity.

Table 1 | Coefficients of variation of all L-Measure derived morphometric features.

\begin{tabular}{ll}
\hline Morphometric features & \multicolumn{2}{c}{ CV for Dendrites } \\
\cline { 2 - 3 } & Hierarchy groups Cluster groups
\end{tabular}

\begin{tabular}{|c|c|c|}
\hline \multicolumn{3}{|l|}{ I. WHOLE TREE/NEURON SIZE } \\
\hline Summed total arbor length & 1.38 & 0.57 \\
\hline Number of arbor tips & 1.65 & 1.82 \\
\hline Total arbor width & 0.68 & 0.43 \\
\hline Total arbor height & 0.65 & 0.51 \\
\hline Total arbor depth & 1.12 & 0.65 \\
\hline \multicolumn{3}{|l|}{ II. BIFURCATION MEASURES } \\
\hline Avg. partition asymmetry & 0.27 & 0.26 \\
\hline Avg. local amplitude angle & 0.17 & 0.17 \\
\hline Max. local amplitude angle & 0.19 & 0.18 \\
\hline Avg. remote amplitude angle & 0.21 & 0.18 \\
\hline Max. remote amplitude angle & 0.24 & 0.23 \\
\hline Avg. local tilt angle & 0.14 & 0.13 \\
\hline Max. local tilt angle & 0.08 & 0.08 \\
\hline Avg. remote tilt angle & 0.09 & 0.08 \\
\hline Max. remote tilt angle & 0.05 & 0.05 \\
\hline Avg. local torque angle & 0.17 & 0.16 \\
\hline Max. local torque angle & 0.11 & 0.11 \\
\hline Avg. remote torque angle & 0.18 & 0.17 \\
\hline Max. remote torque angle & 0.10 & 0.10 \\
\hline \multicolumn{3}{|l|}{ III. BRANCH MEASURES } \\
\hline Avg. tortuosity & 0.08 & 0.07 \\
\hline Avg. fractal dimension & 0.03 & 0.02 \\
\hline Max. fractal dimension & 0.15 & 0.14 \\
\hline Avg. branch path length & 0.59 & 0.41 \\
\hline Max. branch path length & 0.81 & 0.53 \\
\hline \multicolumn{3}{|l|}{ IV. COMPARTMENT MEASURES } \\
\hline Max. branch order & 0.85 & 0.85 \\
\hline Avg. terminal degree & 0.71 & 0.68 \\
\hline Max. path distance from soma & 0.76 & 0.57 \\
\hline Max. branch helicity & 0.19 & 0.16 \\
\hline
\end{tabular}

A detailed description of each metric is provided at http:// cng.gmu.edu:8080/ $\mathrm{Lm} / \mathrm{help} /$ index.htm. 


\section{PRINCIPAL COMPONENT ANALYSIS (PCA) AND CLUSTER ANALYSIS}

In order to reduce the dimensionality of the morphometric space for unsupervised clustering, PCA was run on the feature dataset using the "prcomp" routine in R (v. 2.15.1). This transformation rotates all extracted measures (27 features for 5,099 arbors) such that the first dimensions in the new space capture the most variance (in decreasing order). Prior to PCA, all features were normalized by their respective standard deviations, and the features with absolute skewness greater than unity (17/27) were log-transformed. Negatively skewed distributions were inverted and distributions with negative values were shifted prior to logtransformation. These steps ensure an approximately normal distribution of the input features, as assumed by PCA and subsequent clustering. The resulting first 17 components, accounting for $95 \%$ of the variance, were retained for cluster analysis.

Next, the dendritic arbors were clustered based on their principal morphometric components to seek a shape-based classification independent of a priori metadata grouping. We selected a model-based approach, in which mixtures of Gaussians are found that together have maximal likelihood to fit the data. A cluster is the collection of arbors that are most likely to come from the same multivariate Gaussian (referred to as a cluster model). We used the R “MCLUST" package (Farley and Raftery, 2006) for estimating optimal model parameters and selecting the most likely model type given the dataset. The model types include spherical, ellipsoidal (with a diagonal covariance matrix), and ellipsoidal with orientation (indicating correlation between dimensions). This flexibility makes model-based clustering a more suitable choice than other popular methods (e.g., K-means) for analysis of heterogeneous data sets collated from different experiments, labs, and conditions. Not only are clusters not limited to fit spherically symmetric distributions, but also each cluster is allowed to have its own distinct variance, shape, and orientation.

MCLUST implements Expectation Maximization (EM) to select models using the Bayesian information criterion (BIC). The BIC computes the log likelihood of the cluster model, but includes a penalty for the number of parameters weighted by the log of the dataset size. Thus, goodness of fit is balanced against model simplicity according to the following equation, whereby the largest value determines the best model:

$$
\mathrm{BIC}=-2 \cdot \ln \widehat{L}+k \cdot \ln (n)
$$

Here, $\widehat{L}$ is the maximized likelihood computed on the marginal likelihood $\mathrm{P}\left(\mathrm{y} \mid \mathrm{M}_{\mathrm{i}}\right)$ of the candidate model $\mathrm{M}_{\mathrm{i}}$ given the observed data $\mathrm{y}\left(\mathrm{y}_{1}, \ldots \mathrm{y}_{\mathrm{n}}\right) ; k$ is the number of free parameters to be estimated; and $n$ is the number of data points.

The specification of MCLUST model types and parameters is coded by three letters in each of three positions. The three positions represent the model size, shape, and orientation variables, respectively. Letter " $E$ " indicates that the parameters are equivalent across all clusters, "V" signifies variable parameter values, and " $\mathrm{I}$ " denotes that the corresponding parameter is not applicable. For example, "EII" indicates spherical Gaussians (no shape or orientation) with equal size among clusters, which corresponds to the traditional K-means method. Similarly, the "VVV" model type indicates varying size, shape, and orientation parameters.
This latter model was determined by EM to be optimal for the data analyzed here despite its greater BIC cost implied by the larger number of free parameters. Thus, EM provides information theory-derived evidence that the performance of simple uniform spherical (K-means-like) clustering is sub-optimal for the data used in this study.

Cluster distances from the center of coordinates were measured by $\mathrm{Z}$ score to account for relative variance. Pairwise cluster distances were computed as the distances between the corresponding centers normalized by the cluster scatters, which are defined as averaged distance of the cluster points from the respective cluster center (Dunn, 1973). The associations among clusters and metadata groups were assessed using the chi-square test of independence, using the (marginal) frequencies of group and cluster occurrences to calculate the expected association matrix, and computing the Bonferroni-corrected $p$-values of the observed co-occurrences from the standardized residuals.

\section{RESULTS \\ VARIABILITY OF DENDRITIC MORPHOLOGY AND COMPARISON BY METADATA}

To quantify the heterogeneity of the data, we computed the coefficient of variation (CV) for each of the 27 measured features over the entire set of 7,143 neurons as well as over the subset of 5,099 neurons used in cluster analysis (Table 1). Tortuosity, fractal dimension, and tilt angle are the least variable features, with a $\mathrm{CV}$ of less than $10 \%$. In contrast, size measures are the most variable, with a CV close to or greater than unity. This apparent distinction between "local" (branch-level) vs. "global" (neuron-level) features may reflect both the effect of biological constraints (e.g., varying dimensions of different species from insects to human) and experimental conditions (slice vs. wholeanimal preparations). Most other metrics display intermediate CV values.

Dendritic morphologies were then compared across species, cell types, and brain regions. The corresponding metadata information for each reconstruction in NeuroMorpho.Org was organized hierarchically (Figure 3), forming groups with a sufficient number of neurons to enable statistical comparison of the results (at least 55 for species, 300 for brain regions, and 100 for cell types). Groups with fewer reconstructions were combined into "others" together with the reconstructions missing the detailed metadata information at the corresponding level of the hierarchy (marked as "not reported" in NeuroMorpho.Org).

The "leaf" nodes in each of the three metadata hierarchies (12 for species, 14 for brain regions, and 10 for cell types) were compared with a selection of representative morphometric features (Figure 4). In a limited set of cases, individual groups could be distinguished from the rest or from each other. For example, blowfly and cat reconstructions stood out against the neurons of all other species for their large topological asymmetry and $\mathrm{Z}$ span, respectively. The dendritic arbors of magnopyramidal cells tended to have extensive total length but low fractal dimension, whereas granule cells displayed opposite characteristics. At the same time, most groups show extensive overlap of their morphometric variance, preventing firm statistical conclusions. Part of the reason for such broad distributions is likely due to the non-uniform 


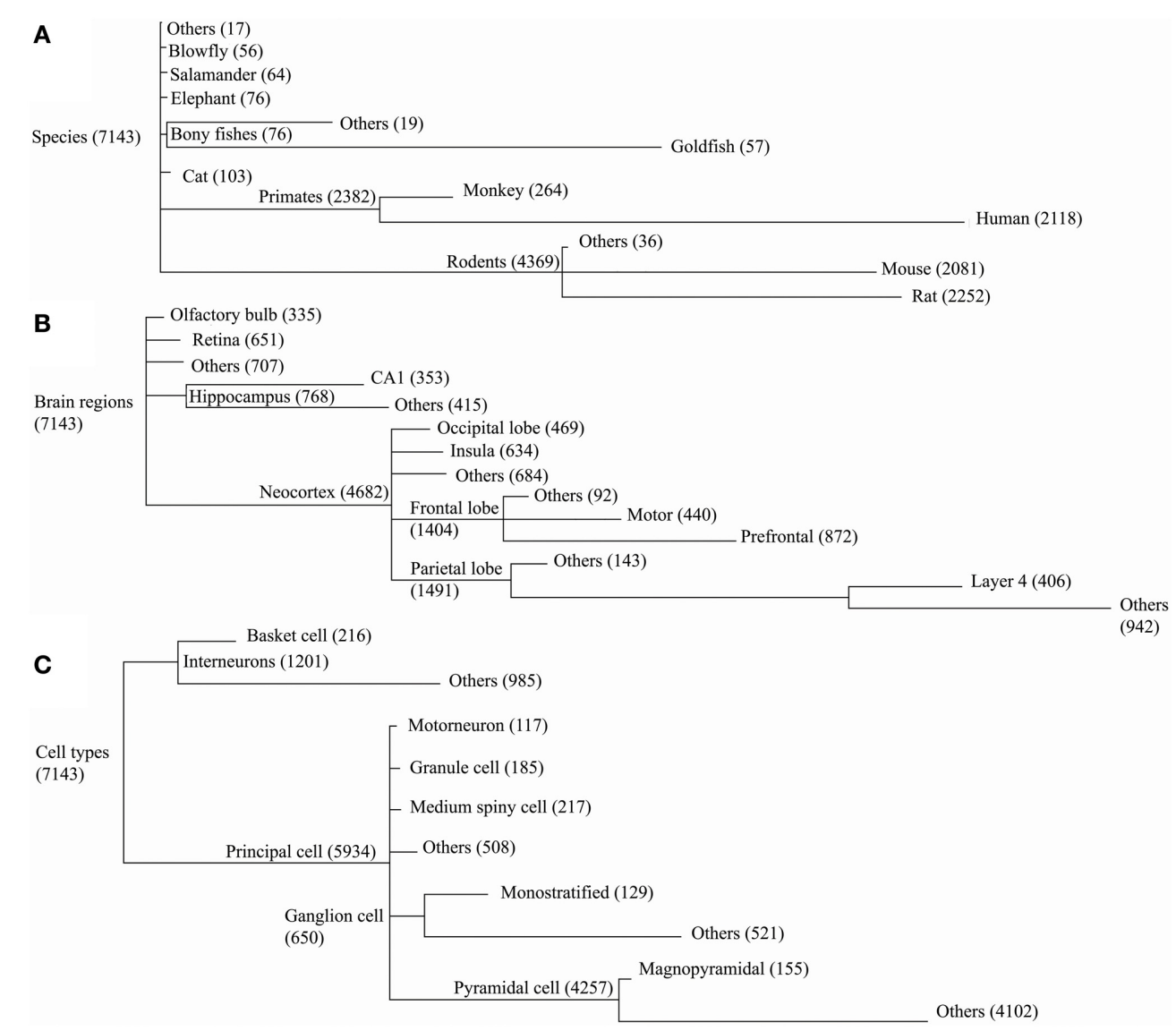

FIGURE 3 | NeuroMorpho.Org v5.6 data is categorized along three major metadata dimensions, namely species, brain regions, and cell types. Reconstructions are hierarchically organized in each of these dimensions. Every node in the hierarchy is labeled by the number of associated reconstructions. The line lengths are proportional to the size of the child nodes relative to their parent nodes. (A) In the species hierarchy nodes with fewer than 55 reconstructions are grouped together with the "not reported" data under "Others." In the brain regions (B) and cell type (C) hierarchies the grouping thresholds are 300 and 100, respectively. nature of archive-wide data sets pooled together across different experiments and laboratories. It is also clear that these metadata dimensions are not mutually independent because of evolutionary constraints (e.g., bony fishes lack a neocortex) and the finite sample of reconstructions (e.g., all monostratified ganglion cells came from the mouse retina). More generally, while popular in comparative anatomy, such a pairwise approach lacks the ability to reveal multivariate effects that are unavoidable given the nonrandom association between metadata groups and experimental conditions.

\section{EXTRACTING PRIMARY MORPHOLOGICAL FEATURES BY PCA AND CLUSTER MODELS}

In order to overcome the above limitations, we adopted an unsupervised clustering approach following dimensionality reduction with PCA. The first step is to reduce the initial parameter space to fewer orthogonal dimensions capturing most of the data variability. In mathematical terms, PCA identifies the linearly independent combinations of variables ordered by the amount of variance they explain. From the (27) original morphometric features, the first 17 dimensions of PCA covered $95 \%$ of the data variance and were used for cluster analysis.

The first 6 of these principal components were responsible for three quarters of the variance and displayed distinctive compositions of their primary morphometric features (Table 2). Identifying the heaviest contributors in the linear combination of morphometric features of each principal component ("loadings") is useful to aid subsequent interpretation of the results. The first component (PC1) is positively loaded on bifurcation angles and negatively on branch path length, reflecting high branching density. The morphometric features most descriptive of PC2 and PC3 are respectively overall size and branch tortuosity. Together, the first three components capture the majority of the data variance. The simplest morphological descriptors of PC4, PC5, and PC6 are arbor flatness (related to torque angle), fractal dimension (or "space filling"), and topological asymmetry (the average normalized sub-tree partition at bifurcation points), respectively.

In order to produce the most informative statistical model, unsupervised clustering selects the optimal number of clusters as well as their parameters, by maximizing the BIC. These data were 

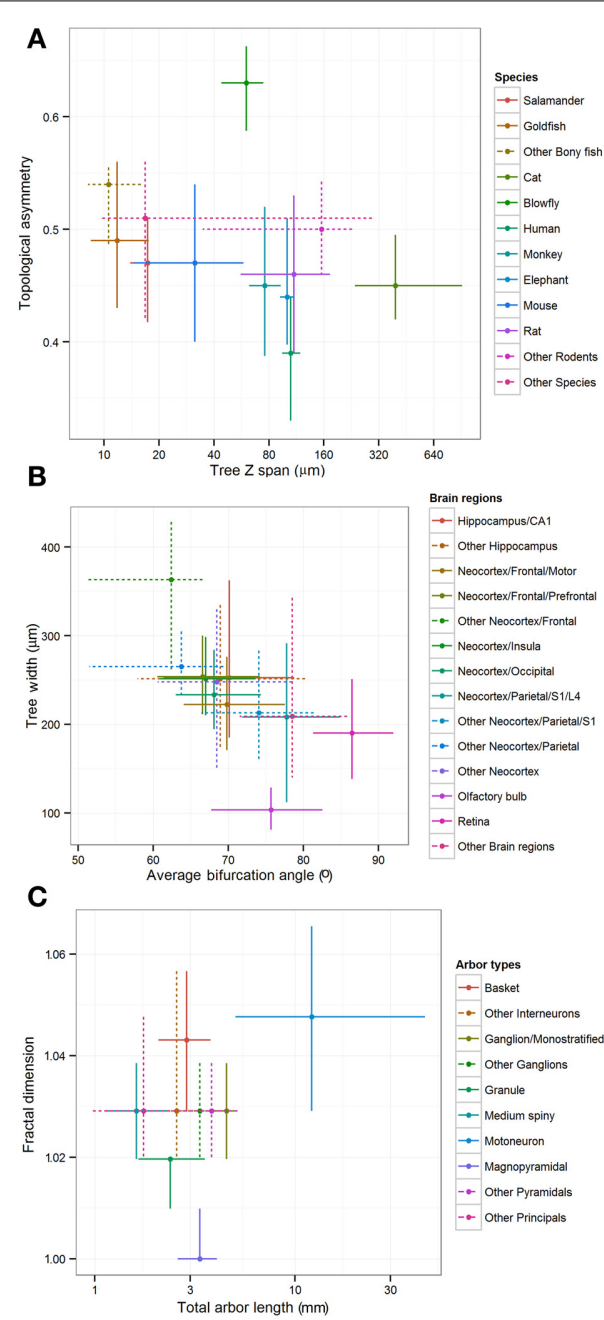

FIGURE 4 | Inter-group differences of representative morphometric features within each main metadata dimension. Crosshairs represent medians and quartile ranges of each group corresponding to the leaf nodes in the hierarchies shown in Figure 3. Dotted lines indicate "other" groups with merged data. (A) Differences in arbor depth and topological asymmetry among species. (B) Differences in arbor width and average bifurcation angle among brain regions. (C) Differences in fractal dimension and total arbor length among cell types.

best fit to six clusters with varying size, shape, and orientation (Figure 5). The numerical difference between this model and the variant with constant cluster shape, however, was minimal (and is undetectable in Figure 5A). The same model type, moreover, performed nearly as well with five or seven clusters as indicated by the absence of a clear peak in the BIC plot. We experimented with these alternative model variant and numbers of clusters and found no substantial differences in findings. At the same time, the data were not adequately described by traditional spherical clusters, even if with unequal sizes (Figure 5A).

Since six clusters correspond to the maximum value for both top model types, we selected this number as the most suitable for exploratory analysis. Such a choice, nevertheless, should not be taken to reflect a ground truth that only six
Table 2 | Primary morphometric loading (with absolute values of $\mathbf{0 . 2 5}$ or higher) of the first six principal components of the dendritic arbors used in cluster analysis.

\begin{tabular}{|c|c|c|}
\hline Principal Component & Morphometric features & Loading \\
\hline \multirow{7}{*}{$\begin{array}{l}\text { PC1 (27\% of cumulative } \\
\text { variance): branching density }\end{array}$} & Max. remote amplitude angle & 0.29 \\
\hline & Avg. remote amplitude angle & 0.27 \\
\hline & Max. local amplitude angle & 0.26 \\
\hline & Avg. terminal degree & 0.25 \\
\hline & Max. branch order & 0.25 \\
\hline & Avg. branch path length & -0.28 \\
\hline & Avg. remote tilt angle & -0.26 \\
\hline \multirow{4}{*}{$\begin{array}{l}\text { PC2 ( } 43 \% \text { of cumulative } \\
\text { variance): size }\end{array}$} & Summed total arbor length & 0.4 \\
\hline & Total arbor height & 0.36 \\
\hline & Max. path distance from soma & 0.34 \\
\hline & Total arbor width & 0.33 \\
\hline \multirow{3}{*}{$\begin{array}{l}\text { PC3 (58\% of cumulative } \\
\text { variance): branch tortuosity }\end{array}$} & Avg. tortuosity & 0.42 \\
\hline & Avg. fractal dimension & 0.34 \\
\hline & Avg. local tilt angle & -0.34 \\
\hline \multirow{2}{*}{$\begin{array}{l}\text { PC4 (64\% of cumulative } \\
\text { variance): arbor flatness }\end{array}$} & Avg. remote torque angle & 0.63 \\
\hline & Avg. local torque angle & 0.62 \\
\hline \multirow{6}{*}{$\begin{array}{l}\text { PC5 ( } 70 \% \text { of cumulative } \\
\text { variance): fractal dimension and } \\
\text { tilt angles }\end{array}$} & Max. fractal dimension & 0.37 \\
\hline & Avg. fractal dimension & 0.35 \\
\hline & Avg. remote tilt angle & 0.35 \\
\hline & Avg. tortuosity & 0.25 \\
\hline & Max. remote tilt angle & -0.32 \\
\hline & Avg. remote amplitude angle & -0.36 \\
\hline \multirow{2}{*}{$\begin{array}{l}\text { PC6 ( } 75 \% \text { of cumulative } \\
\text { variance): partition asymmetry } \\
\text { and depth }\end{array}$} & Avg. partition asymmetry & 0.41 \\
\hline & Total arbor depth & 0.35 \\
\hline
\end{tabular}

"true" classes exist within the data. This selection simply maximizes the inter-similarity of co-clustered classes relative to classes in other clusters given the scope, size, quality, and composition of the available dataset. To determine if further differences exist between classes that associate with the same cluster, it would be appropriate to run the same analysis on a subset of the data (sub-clustering). This additional analysis, however, requires larger datasets to meet the selection criteria based on a minimum number of reconstructions in each dataset.

The two-dimensional data projection on the first and second components illustrates the relative discrimination of clusters by branching density and arbor size (Figure 5B). Cluster ranking by variance-normalized distance from the center of coordinates (Figure 5C) allows for focused analysis on clusters farther from the origin $(a-d)$, and thus morphologically distinctive, relative to those closer ( $e$ and $f$ ) to the origin. The six clusters contain respectively $585(a), 1488(b), 762(c), 555(d), 818(e)$, and $891(f)$ reconstructions. Pairwise distances (Figure 5D) reveal that one and the same cluster $(b)$ is both the farthest from $(a)$ and closest to $(e)$ than to other clusters. 


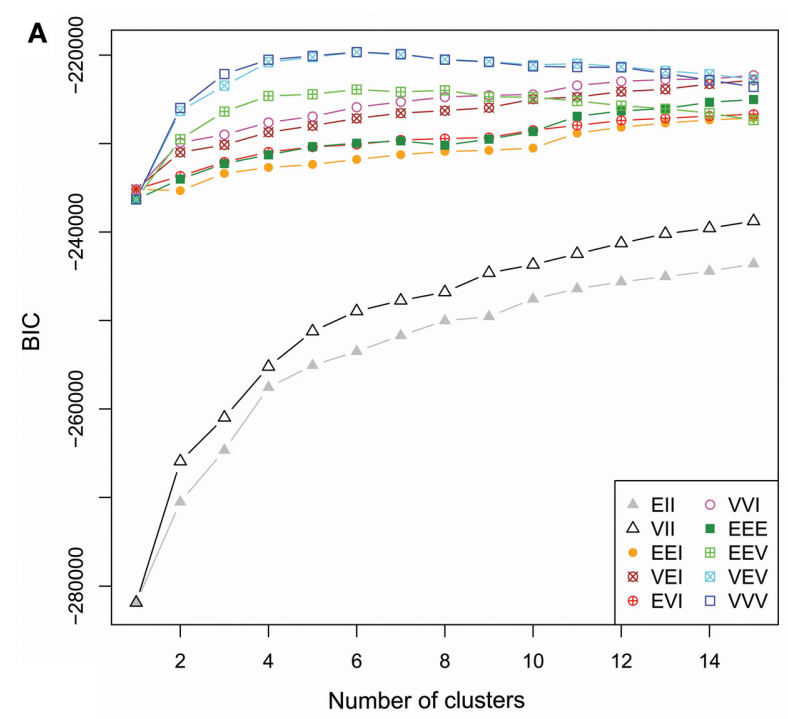

C

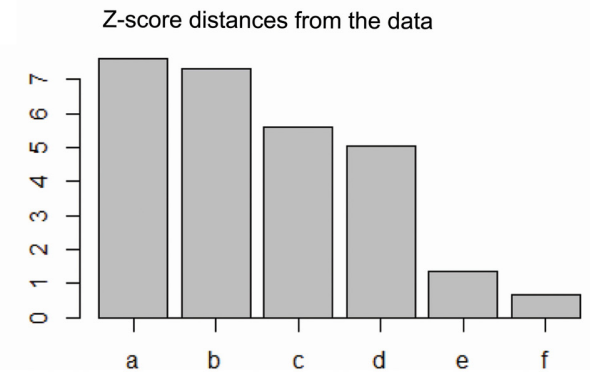

FIGURE 5 | Unsupervised cluster analysis of dendritic morphology. (A) Maximization of $B I C$ reveals marginal performance of spherical clustering with equal (EII) or unequal size (VII) alike relative to the models allowing ellipsoidal clusters. Among those, those accounting for unequal orientation (EEV, VEV, and WV) performed better, especially with unequal size (VEV and VVV). The highest BIC value was attained at 6 clusters with varying size,

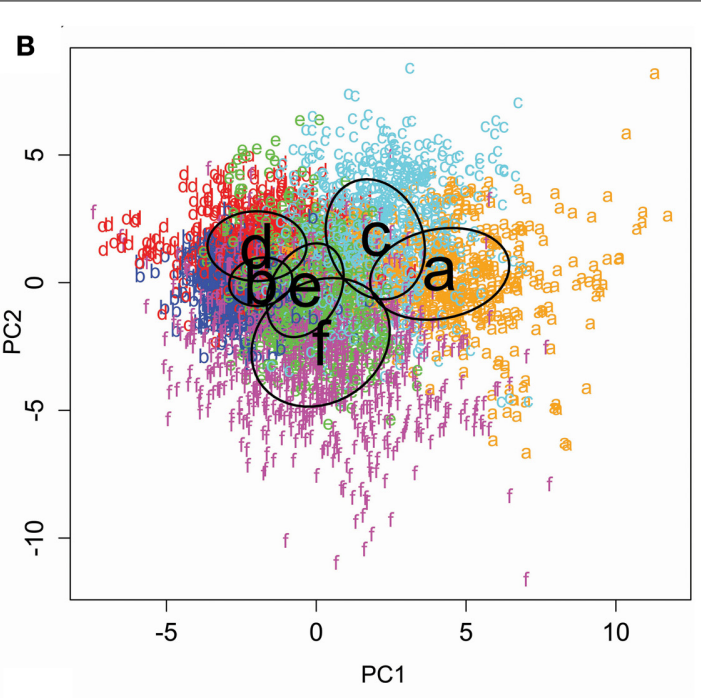

D

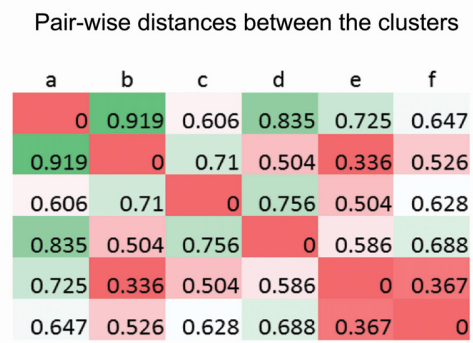

shapes and orientation (VWV). (B) Scatter plot of color-coded cluster assignments ( $a$ through $f$ ) projected on the first two principal components. The ovals represent best fitting cluster parameters. (C) Cluster ranking by $Z$ score distance from the origin of coordinates. (D) Pairwise inter-cluster distances normalized by the corresponding scatters. Farthest distances are in green and nearest are in red.

\section{STATISTICAL ASSOCIATIONS BETWEEN CLUSTERS AND METADATA COMBINATIONS}

Unsupervised cluster models segregate neuronal reconstructions solely based on morphological features. This classification is thus complementary to, and independent of, the metadata associated with each reconstruction. The correspondence between the six morphological clusters and the 45 unique metadata groups characterized by species, brain region, neuron type, and lab of origin can shed light on the most important morphometric signatures of each metadata group. The 45 -by- 6 chisquare contingency matrix (Table 3 ) reports the probabilities that the observed over-representation and under-representations of associations between morphological clusters and metadata groups would be due to chance if the observed numerical compositions of each cluster and group were independent of each other. For example (first data row in Table 3), pyramidal neurons from mouse primary somatosensory cortex in SmitRigter's archive are significantly over-represented in cluster $a$ $\left(p<0.0002=10^{-3.73}\right)$ and significantly under-represented in cluster $b\left(p<0.001=10^{-3.05}\right)$. In contrast, the proportion of these same neurons in cluster $d$ is within the range expected from the sizes of this metadata group and morphological cluster.

Interestingly, each and every metadata group is overrepresented in, and thus associated with, one of the six morphological clusters. The majority (38/45) are associated with exactly one cluster, and all of the remaining (7/45) are each split between just two clusters. Most possible metadata/cluster pairs deviated significantly from the random distribution expected from the "null hypothesis": 53 out of 270 were significantly overrepresented and 87 out of 270 significantly under-represented. This overall partition of metadata groups in distinct clusters constitutes a remarkable outcome for a fully unsupervised classification method. Certain metadata groups are over-represented in one morphological cluster and under-represented in all other clusters, such as ganglion cells from mouse retina in Masland's archive (cluster $a$ ) and pyramidal cells from human prefrontal cortex in Jacobs' archive (cluster $b$ ). Other metadata groups are over-represented in one morphological cluster, but otherwise scattered throughout all other clusters per the respective numerical abundance, such as pyramidal cells from monkey frontal lobe in Luebke's archive (cluster $d$ ) 
Table 3 | Matrix of positive (green) and negative (red) associations between metadata groups (rows) and morphological clusters (columns).

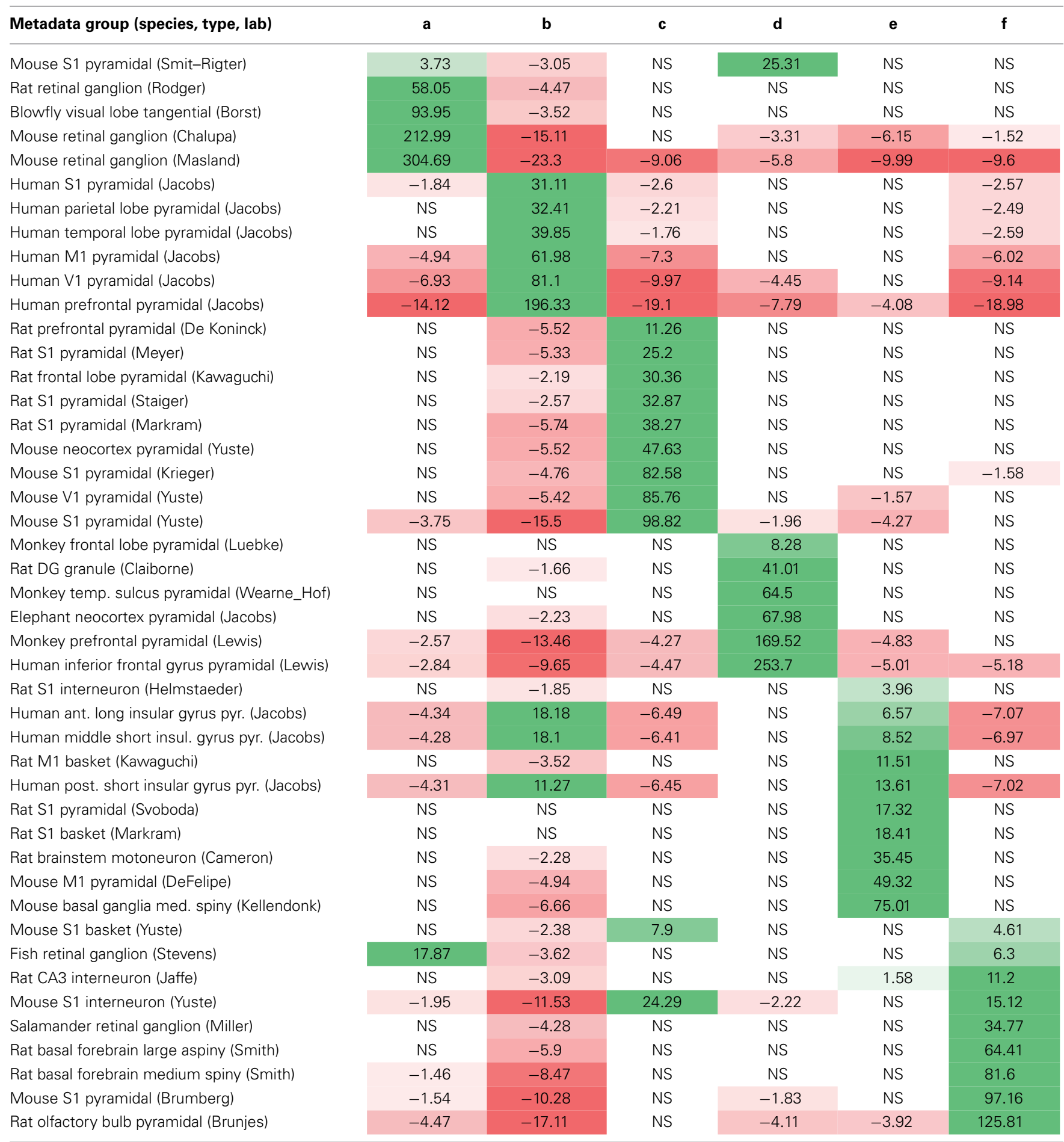

The Bonferroni adjusted p-values obtained by the chi-square test of independence are converted for ease of comparison into log 10 values, inverting the sign for overrepresented (green) cells. The color gradient shows the interaction strength. Non-significant $(p>0.05)$ associations are indicated with NS.

and motoneurons from rat brainstem in Cameron's archive (cluster $e$ ).

Several observations can be made that transcend individual archive identities. All rodent retinal ganglion cell groups are associated with cluster $a$, whereas fish and salamander retinal ganglion cells groups are associated with cluster $f$. The relative cluster positions in the first two principal components and the corresponding morphological loadings (Figure 5B and Table 2) suggest that the retinal ganglion cells are larger and with denser branching in rodents than in non-mammals. Neocortex pyramidal cell groups 
are distributed across all clusters, with preference mostly based on species (most notably, human in $b$, rodents in $c$, and monkey in $d$ ). All rodent non-cortical and non-pyramidal cell groups are found in cluster $f$ (along with salamander and fish retinal ganglion cells). Such metadata heterogeneity, together with this cluster's minimal distance from the morphological center (Figure 5C) suggests a putative "catch-all" role for cluster $f$, which makes it broadly representative of the whole dataset.

In several cases, the split of a metadata group into two morphological clusters reflects previously reported relations. For example, three groups of pyramidal neurons from the (anterior, middle, and posterior) human insular gyrus in the Jacobs' archive divided between clusters $b$ and $e$ according to structural differences related to the subject's gender (Anderson et al., 2009). Similarly, mouse primary somatosensory pyramidal cells are overrepresented in both clusters $a$ and $d$, consistent with the reported differences between young and adult animals (Smit-Rigter et al., 2012). The grouping of neurons from younger mice with retinal ganglion cells (in cluster $a$ ) and from the older mice with pyramidal cells of larger mammals, such as monkey, elephant, and human (in cluster $d$ ), could be expected since the former groups are characterized by the shortest branch path length and the latter groups by the largest. The scattered clustering of pyramidal neurons, however, does not necessarily reflect existing biological relations, but might rather result from the combination of the choice of analysis algorithms, selection of parameters, and experimental differences.

The other splits of metadata groups between two clusters (Table 3) similarly revealed differences likely due to experimental procedures, such as staining protocol or slicing direction, which were not recognized in the original reports (Anderson et al., 1995; Soloway et al., 2002; Goldberg et al., 2003; MacLean et al., 2005; Nikolenko et al., 2007; Woodruff et al., 2009). For example, the separate clustering of different mouse S1 pyramidal cell datasets can be explained by the differences between intracellular biocytin injection (e.g., Yuste's archive) and bulk Golgi staining (e.g., Brumberg's archive). While the mechanisms underlying the different visualization by these techniques are not yet fully understood (Thomson and Armstrong, 2011), the histological labeling information is available as metadata in NeuroMorpho.Org, thus aiding interpretation.

A complementary way to examine the associations between morphological clusters and metadata groups is to systematically analyze the composition of each cluster in terms of its associated groups, broken down by fraction of group, fraction of cluster, and neuron count (Table 4). For example (first data row in Table 4), $33 \%$ of the mouse S1 pyramidal cells from the Smit-Rigter archive are in cluster $a$, accounting for only $3 \%$ of this cluster (17 out of 560 neurons). The sums of cluster fractions in Table 4 correspond to the proportion of neurons in each cluster (e.g., 97\% for cluster $a$ ) made up by the cluster's associated metadata groups (green entries in Table 3). The remaining portions of the clusters are composed of neurons falling outside of their associated cluster. Notably, the blowfly tangential cell group is associated with cluster $a$. Moreover, clusters $b$ and $c$ are exclusively associated with human pyramidal cell (in which only basal dendrites are reconstructed) and rodent neocortex cell groups respectively.

\section{PAIRWISE MORPHOMETRIC COMPARISONS OF NEURON GROUPS IDENTIFIED BY CLUSTER ANALYSIS}

Exploratory inspection of neuronal clusters in the 6-dimensional space of principal morphometric components together with the association between clusters and metadata groups (Tables 3, 4) suggested closer inspection of specific morphological features in selected pairs of neuronal groups defined by their species, brain region, and cell type. The first example pertains to rodent retinal ganglion cells (Figure 6), which are characterized by high branching density and related morphological features (e.g., wide bifurcation angles). These neurons, pooled from mice and rats in four different archives, constitute $80 \%$ of cluster $a$, the farthest away from the center (Figure 5C and Table 4). At the opposite end along the first principal components is cluster $b$, entirely made of human pyramidal basal dendrites. Visual inspection (Figure 6B) reveals the distinctive shapes of rodent ganglion cells and human basal dendrites. Statistical analysis of the two main morphological loadings of PC1 (bifurcation amplitude and branch path length) confirmed the considerable difference between these two neuron groups, even when including those found in clusters other than $a$ and $b$ (Figure 6C).

The second most prominent group in cluster $a$ is constituted by blowfly tangential sensory neurons. These neurons share with the rodent ganglion cells not only comparable branching density properties captured by PC1 (low branch path length and high bifurcation angle), but also similar distributions on PC2 through PC5 and all corresponding morphological features loading on those dimensions. These include measures of size (e.g., total dendritic length and spanned volume), of space filling (fractal dimension and tortuosity), and of arbor planarity (torque and tilt angles). Such tight alignment on the first five principal components along with the morphological co-clustering suggests a structural basis for the functional commonalities between blowfly tangential cells and retinal ganglion cells, both of which process motion-sensitive visual information (Kong et al., 2005; Cuntz et al., 2008).

Nevertheless, rotation on the sixth principal component exposed a surprising and nearly perfect separation between retinal ganglion cells and blowfly tangential cell (Figure 6A). Since the main morphological feature loading on PC6 is topological asymmetry (the average partition of terminal degree over all bifurcations), we compared the distribution of this measure between the two neuron classes (Figure 6D). This analysis demonstrated that blowfly tangential neurons have much more asymmetric bifurcations than ganglion cells (and most typical neurons). Interestingly, the data projection over the first and sixth principal components (Figure 6A) also suggested a linear relationship between topological asymmetry and branching density in rodent retinal ganglion cells but not in other groups. The Pearson correlation coefficients for branching density and asymmetry index $(R=-0.50)$ and for bifurcation amplitude remote and asymmetry $(R=0.51)$ are both statistically highly significant $\left(p<10^{-10}\right)$.

Rotating the data along the first and third principal components (related to branching density and tortuosity, respectively) revealed another distinct relationship across pyramidal cells from different species, brain regions, and developmental 
Table 4 | Composition of the six morphological clusters in terms of their over-represented metadata groups.

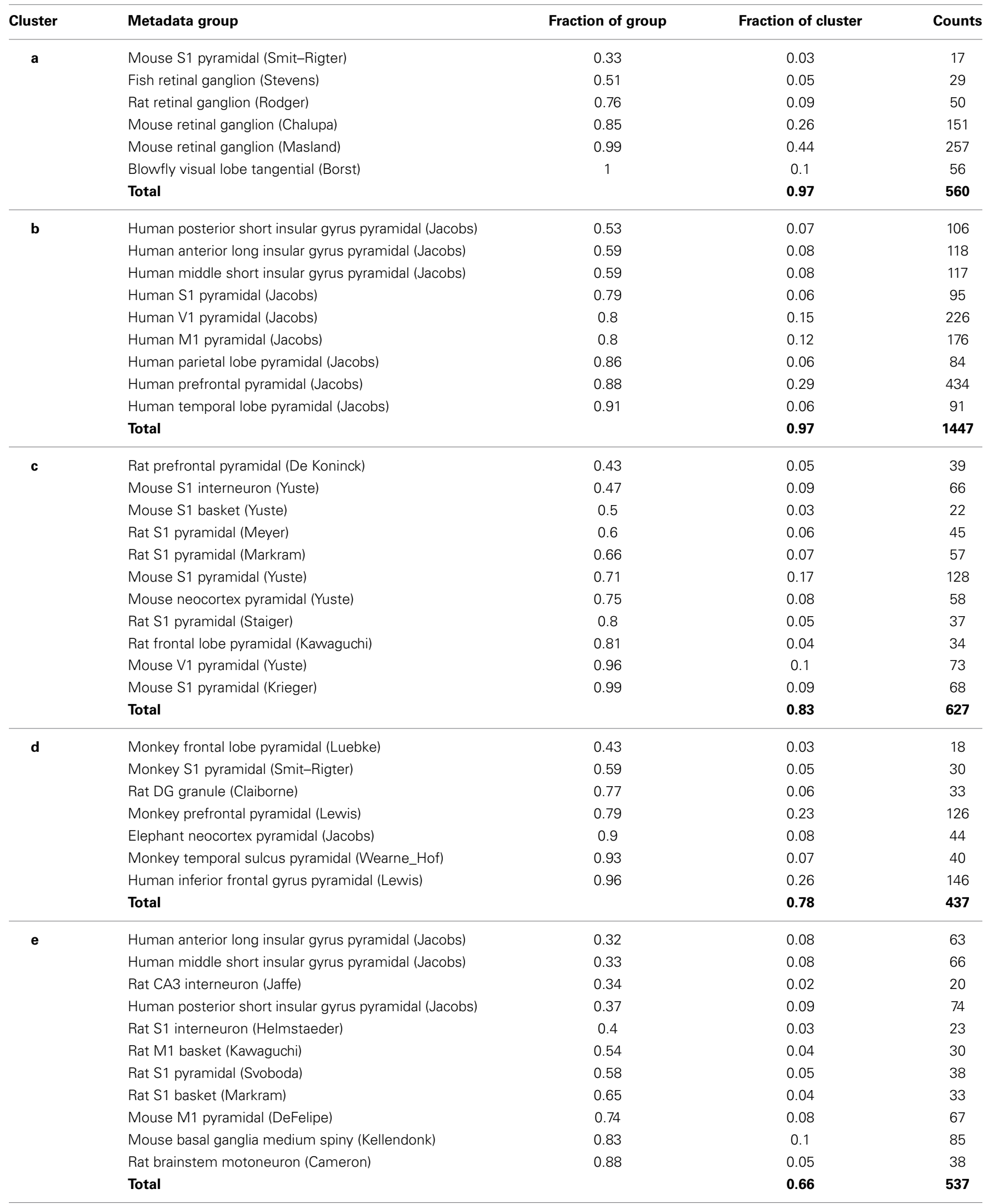


Table 4 | Continued

\begin{tabular}{|c|c|c|c|c|}
\hline Cluster & Metadata group & Fraction of group & Fraction of cluster & Counts \\
\hline & Fish retinal ganglion (Stevens) & 0.47 & 0.03 & 27 \\
\hline & Rat CA3 interneuron (Jaffe) & 0.55 & 0.04 & 32 \\
\hline & Salamander retinal ganglion (Miller) & 0.78 & 0.06 & 50 \\
\hline & Rat olfactory bulb pyramidal (Brunjes) & 0.8 & 0.18 & 164 \\
\hline & Rat basal forebrain medium spiny (Smith) & 0.88 & 0.11 & 95 \\
\hline & Rat basal forebrain large aspiny (Smith) & 0.9 & 0.08 & 73 \\
\hline & Total & & 0.72 & 637 \\
\hline
\end{tabular}

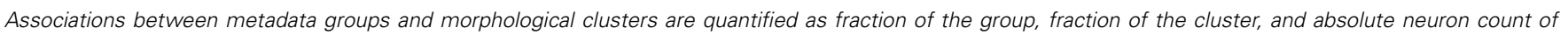
group/cluster intersection. Within cluster, groups are arranged in ascending order of the group fraction.

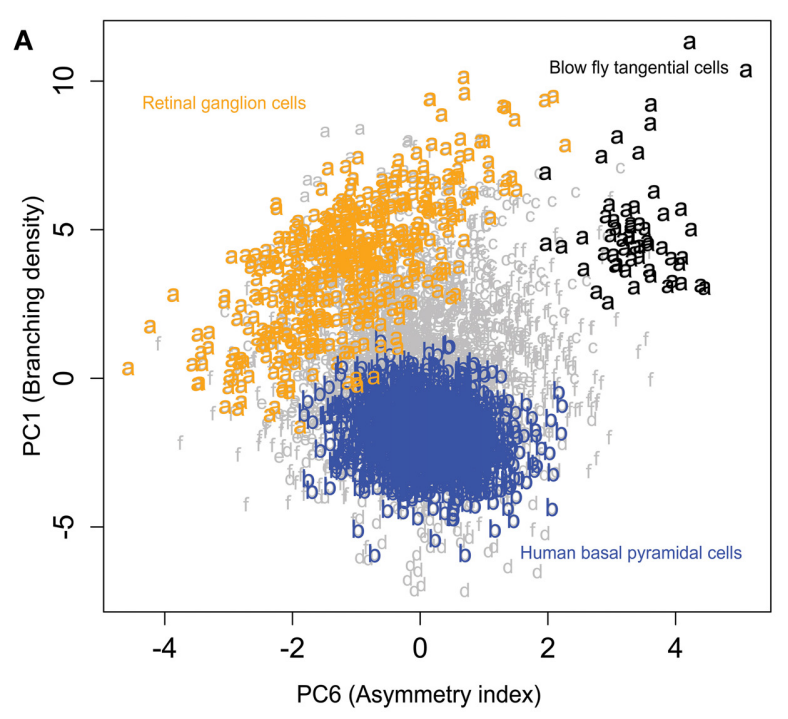

C

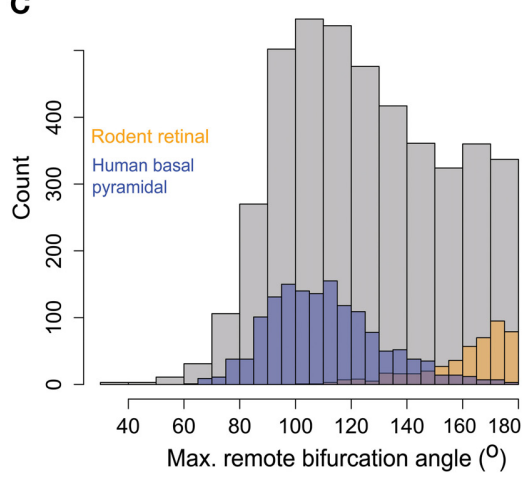

D

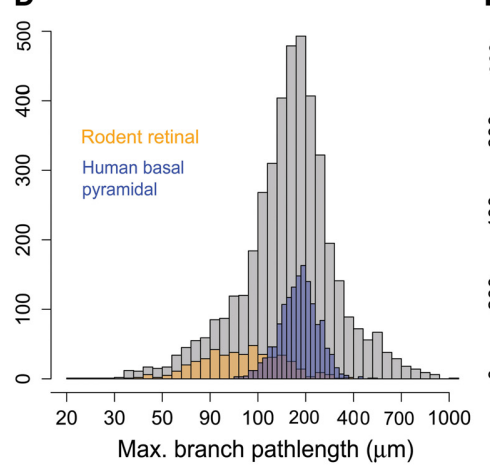

B
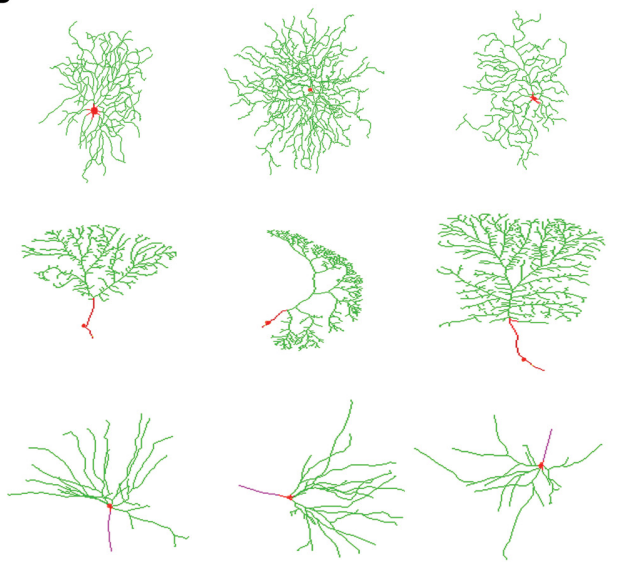

$\mathbf{E}$

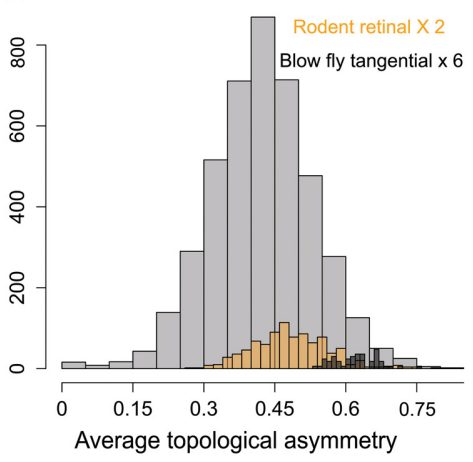

FIGURE 6 | Similarities and differences of rodent retinal ganglion cells with other neurons within and across clusters. (A) All rodent retinal ganglion cells together with blowfly tangential cells (cluster a) show highest branching density along PC1 compared to others metadata groups. The human basal pyramidal cell cluster (b) is highlighted for comparison. PC6 separates the tangential and ganglion cells, showing sub-cluster differences. The retinal cells also show a pattern of increasing partition asymmetry with increasing branching density. (B) Sample images of retinal ganglion cells (top), blowfly tangential cells (middle), and human basal pyramidal cells (bottom). NeuroMorpho. Org IDs of these neurons from left to right: 06464, 05352, 05405, 06652, 01895, 06640, 03723, 03724, 03722. (C) Rodent ganglion cells have larger amplitude angles compared to human basal pyramidal cells (and most other cell classes). (D) Rodent ganglion cells also display shorter branch length, corresponding to higher branching density. (E) The blowfly neurons, while sharing similar branch path length and amplitude angles with the retinal cells, have higher topological asymmetry. 
stages (Figure 7). Specifically, neocortical pyramidal cells from rodents (clusters $c$ ) and primates (cluster $d$ ) display a trend of increasing branch tortuosity with increasing branch density (Figure 7A). Visual examination of morphologies selected from the corresponding clusters in the PC1-PC3 scatter plot demonstrates a correspondence in the increase of branch density and branch tortuosity (Figure 7B). The least tortuous trees, and many of the primate neurons, are noted to be incomplete reconstructions, in which only dendrites proximal to the soma are traced. In contrast, the dendrites of rodent neocortical pyramidal neurons tend to be fully reconstructed in both apical and basal arbors.

\section{CRITICAL ASSESSMENT OF POTENTIAL CONFOUNDS}

In the course of the iterative process of data inspection, hypothesis formulation, research design, and quantitative analysis, we encountered numerous challenges pertaining to data validation, curation, and standardization across labs. After a preliminary exploration of the entire content of NeuroMorpho.org, we decided to include in our study only approximately half of the available neurons. Specifically, we chose to avoid multi-lab analysis of axons, because of the extreme dependence of axonal morphology on experimental conditions. In our early analysis attempt that did not segregate axons from dendrites, biological findings became practically impossible to disentangle from major artifacts. This selection effectively defines a standard of minimal requirements for effectively comparing neural arbors.

Moreover, we excluded measures related to branch diameter (branch power ratios, surface areas, occupied volume, etc.) due to their strong sensitivity on the inter-laboratory variety of labeling or staining, imaging resolution or optical magnification, and other experimental details affecting tracing conditions (Scorcioni et al., 2004). Furthermore, most reconstructed cells originate from preparations in acute brain slices (in vitro). In the primary somatosensory region of rat neocortex (S1), this common preparation may result in trimming off more than $50 \%$ of the dendritic arbor (Oberlaender et al., 2012). These slicing artifacts impact larger brains to a greater extent, as reflected by the fact that human cells are only represented by basal dendrites. In addition to species differences, trimming effects also depend on animal age, slicing thickness and orientation, and the depth of electrode penetration in the tissue. For these reasons, when mining the cluster analysis results, we paid particular attention to only report findings as "biological" (Figures 6,7) that were not based on size or any morphometrics significantly affected by trimming artifacts. Instead, we identified correlations based on measures such as branching density, tortuosity, and branch angles, all of which have been previously found to be consistent between in vitro and in vivo preparations (Pyapali et al., 1998).

On the one hand, this judicious design allowed the independent reproduction of findings reported in prior publications. These included several cases of "split metadata groups" into two morphological clusters, which reflected structural differences related to the subject's gender (Anderson et al., 2009) or developmental stage (Smit-Rigter et al., 2012). On the other hand, experimental artifacts still contributed to clustering, and other splits of metadata groups between two clusters (Table 3 ) revealed differences likely due to staining protocol or slicing direction, which were not recognized or discussed in the original reports (Anderson et al., 1995; Soloway et al., 2002; Goldberg et al., 2003; MacLean et al., 2005; Nikolenko et al., 2007; Woodruff et al., 2009). Thus, database-wide analyses can reveal potential confounds not easily pinpointed in individual studies.

One of the most common artifacts of tissue processing is shrinkage, and this factor is also highly variable among labs. Shrinkage differentially affects the slice planar and perpendicular dimensions (the latter typically producing a larger effect).

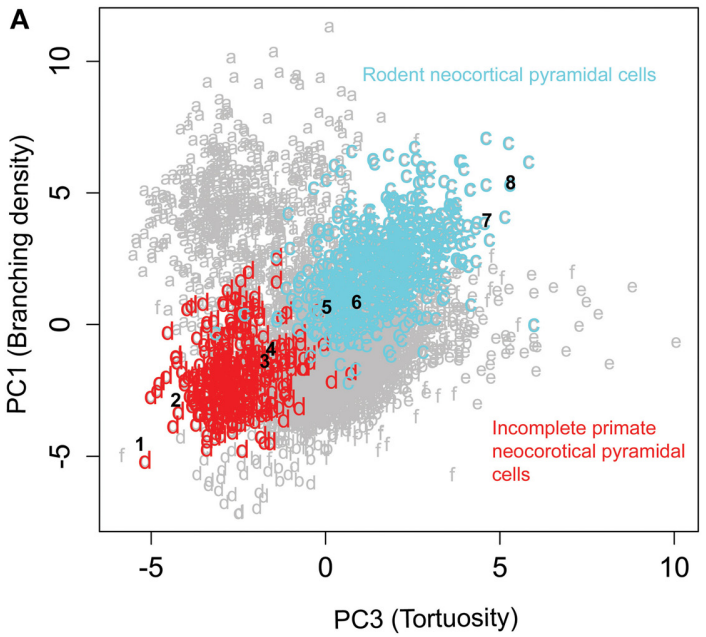

FIGURE 7 | Rodent and primate cortical pyramidal cells show a distinct linear relationship between PC1 and PC3. (A) The majority $(71 \%)$ of cluster $c$ consists of rodent cortical pyramidal cells, whereas a similar proportion of cluster $d(72 \%)$ corresponds to primate pyramidal cells, which tend to be only partially reconstructed. (B) Sample images of incomplete primate pyramidal
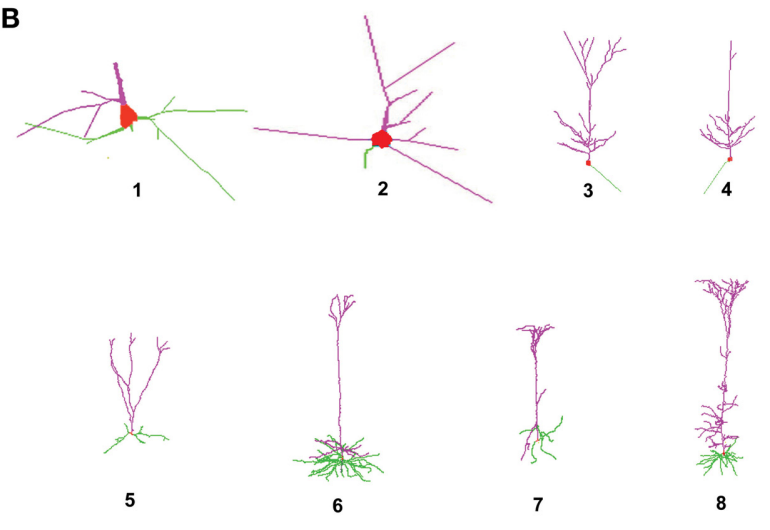

cells in the top row (1-4) and rodent cortical pyramidal cells in the bottom (5-8). The numbers indicate their corresponding position in the cluster plot illustrating the progressive increase in branching density and tortuosity in both clusters. The NeuroMorpho. Org IDs of these neurons from left to right: 01821, 01526, 01627, 01623, 09630, 09474, 02569, 00266. 
Thinner slices tend to shrink more and so do preparations from younger animals. The duration of the experimental procedure may also impact shrinkage, as do the bathing and embedding media. Shrinkage can be measured in all dimensions and it can therefore be compensated for by multiplying the resulting position coordinates by an appropriate correction factor. However, this post-processing operation also exacerbates noise due to light diffraction and other experimental errors. These sources of errors tend to be larger in the direction corresponding to the depth of the slice (" $Z$ "), which is usually estimated through a piezocontroller in the motorized stage. Moreover, shrinkage typically varies both within and between sections, and an accurate calibration therefore requires multiple repeated measurements that add to the already demanding labor intensity of digital reconstruction. For these reasons, shrinkage is not always measured, reported or corrected for. This variability across published studies further worsens the numerous sources of differences due to experimental processing.

In light of the above consideration, we specifically looked for potential shrinkage-related confounds in the clustering results. Out of 56 unique combinations of clusters, metadata groups, and corresponding published articles, only 14 reported shrinkage estimates or mentioned shrinkage altogether. Of those, a mere 5 applied the corresponding correction to the data. Unsurprisingly given the limited sample, we found no statistically significant association between both corrected or uncorrected values and clustering. Next, we examined slicing thickness, which was reported in 49 (out of 56) cases (with median $200 \mu \mathrm{m}$ ). Values varied broadly from 80 to $400 \mu \mathrm{m}$, with $85 \%$ of them falling between 120 and $350 \mu \mathrm{m}$. No statistical association was found between clustering and these values. The lack of explicit shrinkage information prevents firm conclusions and leaves open the possibility that some of the findings we report may be ultimately due to slicing artifacts. However, the low coefficient of variation of measurements typically sensitive to shrinkage, especially tortuosity and fractal dimension (Table 1), suggests that the noise related to shrinkage (as opposed to that affecting size measures) may affect most of the analyzed data to a similar degree.

Fully assessing the potential usefulness of the reported results will require additional investigation. For example, morphologically detailed electrophysiological simulations might be useful to explore how the observed relations between datasets (Figure 6) or between morphological variables (Figure 7) could affect input/output relationship of individual neurons (e.g., Scorcioni et al., 2004; Komendantov and Ascoli, 2009). Similarly, the effect of these morphological relations on potential network connectivity could be studied by embedding the digital reconstructions in an appropriate three-dimensional model of the surrounding neural tissue (e.g., Chiang et al., 2011; Ropireddy and Ascoli, 2011). The continuous expansion of the available pool of neuronal reconstructions will also enable the future validation and refinement of these results with additional or independent datasets.

\section{DISCUSSION}

This work illustrates how shared morphological data can lead to new observations of potential neurobiological interest by enabling statistical quantification of commonalities and differences among neuron groups. However, our results also demonstrate the challenges of working with large-scale datasets from heterogeneous sources, even after extensive effort in metadata curation and management as well as in data standardization and selection. Direct analysis of selected morphometric features among large neuron groups organized by the main metadata dimensions of species, brain region, and cell type failed to reveal meaningful patterns beyond the well-known variability of neuronal shape. At the same time, systematic pairwise examination of all 45 neuronal groups with distinct species, brain region, cell type, and lab of origin for each of the 27 main morphological features would produce more than 50,000 comparisons, raising questions of scientific interpretation and statistical significance.

To overcome these issues, we adopted principal component analysis to identify the most discriminant morphological features throughout the dataset, and model-based cluster analysis to segregate neuron groups solely on the basis of the morphometric characteristics. This approach allowed rigorous examination of the statistical associations between clusters and metadata and inspection of the most informative morphological measurements on the basis of their principal component loadings. The results revealed morphological differences between specific cell types and animal species that were robust to lab provenance while retaining considerable sensitivity to developmental stages and fine regional location as well as to the original experimental conditions. For example, neocortical pyramidal cells from rodents and primates alike display a trend of increasing branch tortuosity with increasing branch density (Figure 7A). This distinct relationship, holding across different species, brain regions, and developmental stages, appears robust to slicing artifacts as demonstrated by the co-alignment of both partially reconstructed and fully reconstructed neurons (Figure 7B).

The primary features of dendritic morphology corresponded to branching density, size, space filling, and bifurcation asymmetry. Of these features, size is likely to be the most dramatically impacted by differential trimming artifacts from brains of varying size. Nevertheless, the most interesting biological findings were based on branch- or bifurcation-level observations. Rodent retinal ganglion cells stood out for their extreme branching density, and clustered together with other neuron types involved in primary sensory processing as well as with developing pyramidal cells from the somatosensory cortex of 6-9 day-old rat. Moreover, the results also highlighted species differences within the same cell types by differentiating retinal cells of rodent from those of fish and amphibians. Specifically, ganglion cells have denser branching and wider bifurcation angles in rodents than in nonmammalian vertebrates (Figures 5B, 6, Table 2). This observation is based on pooling of mice and rats data from four different labs in one cluster, and of fish and salamander from two different labs in the other, and we failed to find any methodological reasons that could explain these morphological differences.

Blowfly tangential sensory neurons are similar to the rodent ganglion cells in many morphological features (e.g., low branch path length, comparable fractal dimension, tortuosity, and arbor planarity), possibly providing a geometric correlate for their similar function in processing motion-sensitive visual information 
(Kong et al., 2005; Cuntz et al., 2008). Nevertheless, retinal ganglion cells and blowfly tangential cells can also be neatly distinguished due to the much more asymmetric bifurcations of the latter neurons (Figure 6A) relative to those of the former (and of most typical neurons). Interestingly, cluster analysis also suggested a linear relationship between topological asymmetry and branching density in rodent retinal ganglion cells but not in other groups, pointing to a previously unrecognized peculiar morphological signature of this class only.

The branching density of mature cortical pyramidal cells, in contrast, was at the opposite end relative to ganglion cells (also demonstrating the effect of developmental changes) and displayed a distinctive correlation with branch tortuosity. Adult neocortex pyramidal cells represent the largest population in NeuroMorpho.Org and come from a broad range of animals, anatomical subregions, layers, and experimental conditions, enabling certain morphological differentiations (e.g., rodent S1 vs. primate M1). Non-cortical neurons, including striatal, olfactory, and others, were distinguished for the smaller size and larger variability of their dendritic arbors.

Several recent investigations have adopted similar analysis designs and strategies for dimensionality reduction, mainly for the purpose of exploratory neuron type classification (e.g., Kong et al., 2005; McGarry et al., 2010; Santana et al., 2013). Alternative approaches to develop automated machine-learning classifiers for identifying neuron types also promise to be effective for large data sets. The present exploratory study used multivariate morphometric analysis to identify the most informative morphological features that distinguish between neuron groups organized by their metadata. We predict that statistical morphometric mining will also prove to be useful for developing quantitative hypotheses and for designing computational models of dendritic growth. At the same time, we discussed the considerable challenge of pooling together data from disparate experimental conditions, and the resulting analysis limitations.

Generation of standardized morphological data across laboratories and research designs could yield much more powerful large-scale data mining. In particular, we are convinced that better clustering would result from more consistent data collection. Systematic reliability assessment of experimental protocols can maximize morphological reproducibility and minimize tracing artifacts (e.g., Dercksen et al., 2014). Any such improvements would also help refine cluster analysis by reducing variability. Unfortunately, the arguably "ideal" experimental choices (in vivo labeling, reconstructions at the resolution limit of light, systematic measurement and compensation of tissue shrinkage, serial tracing across histological sections, etc.) also correspond to the most labor-intensive conditions for manual or semi-manual morphological reconstructions. This tension between quality, sample size, and research cost underscores the need and desirability of fully automated and broadly applicable tracing technologies (Brown et al., 2011; Donohue and Ascoli, 2011).

\section{ACKNOWLEDGMENTS}

We thank Dr. Diek Wheeler, Dr. Rubén Armañanzas, and Mr. David Hamilton for feedback on an earlier version of the manuscript. This work was supported in part by NIH grant R01
NS39600 and a Keck NAKFI award from the National Academy of Science. Publication of this article was funded in part by the George Mason University Libraries Open Access Publishing Fund.

\section{REFERENCES}

Anderson, K., Brian, B., Brooks, R., Charles, H., Lee, H., Ford, K., et al. (2009). The morphology of supragranular pyramidal neurons in the human insular cortex: a quantitative Golgi study. Cereb. Cortex 19, 2131-2144. doi: 10.1093/cercor/bhn234

Anderson, S. A., Classey, J. D., Condé, F., Lund, J. S., and Lewis, D. A. (1995). Synchronous development of pyramidal neuron dendritic spines and parvalbumin-immunoreactive chandelier neuron axon terminals in layer III of monkey prefrontal cortex. Neuroscience 67, 7-22. doi: 10.1016/03064522(95)00051-J

Ascoli, G. A. (2007). Successes and rewards in sharing digital reconstructions of neuronal morphology. Neuroinformatics 5, 154-160. doi: 10.1007/s12021-0070010-7

Ascoli, G. A., and Krichmar, J. L. (2000). L-neuron: a modeling tool for the efficient generation and parsimonious description of dendritic morphology. Neurocomputing 32, 1003-1011. doi: 10.1016/S0925-2312(00)00272-1

Ascoli, G. A., Krichmar, J. L., Scorcioni, R., Nasuto, S. J., and Senft, S. L. (2001). Computer generation and quantitative morphometric analysis of virtual neurons. Anat. Embryol. 204, 283-301. doi: 10.1007/s004290100201

Briggman, K. L., and Denk, W. (2006). Towards neural circuit reconstruction with volume electron microscopy techniques. Curr. Opin. Neurobiol. 16, 562-570. doi: 10.1016/j.conb.2006.08.010

Brown, K., Sugihara, M. I., Shinoda, Y., and Ascoli, G. A. (2012). Digital morphometry of rat cerebellar climbing fibers reveals distinct branch and bouton types. J. Neurosci. 32, 14670-14684. doi: 10.1523/JNEUROSCI.2018-12.2012

Brown, K. M., Barrionuevo, G., Canty, A. J., De Paola, V., Hirsch, J. A., Jefferis, G. S., et al. (2011). The DIADEM data sets: representative light microscopy images of neuronal morphology to advance automation of digital reconstructions. Neuroinformatics 9, 143-157. doi: 10.1007/s12021-010-9095-5

Burgalossi, A., Herfst, L., von Heimendahl, M., Förste, H., Haskic, K., Schmidt, M., et al. (2011). Microcircuits of functionally identified neurons in the rat medial entorhinal cortex. Neuron 70, 773-786. doi: 10.1016/j.neuron.2011. 04.003

Chiang, A. S., Lin, C. H., Chuang, C. C., Chang, H. M., Hsieh, C. H., Yeh, C. W., et al. (2011). Three-dimensional reconstruction of brain-wide wiring networks in Drosophila at single-cell resolution. Curr. Biol. 21, 1-11. doi: 10.1016/j.cub.2010.11.056

Costa Lda, F., Zawadzki, K., Miazaki, M., Viana, M. P., and Taraskin, S. N. (2010). Unveiling the neuromorphological space. Front. Comput. Neurosci. 4:150. doi: 10.3389/fncom.2010.00150

Cuntz, H., Forstner, F., Haag, J., and Borst, A. (2008). The morphological identity of insect dendrites. PLoS Comput. Biol. 4:e1000251. doi: 10.1371/journal.pcbi.1000251

Cuntz, H., Mathy, A., and Hausser, M. (2012). A scaling law derived from optimal dendritic wiring. Proc. Natl. Acad. Sci. U.S.A. 109, 11014-11018. doi: 10.1073/pnas.1200430109

Dercksen, V. J., Hege, H. C., and Oberlaender, M. (2014). The Filament Editor: an interactive software environment for visualization, proof-editing and analysis of 3D neuron morphology. Neuroinformatics 12, 325-339. doi: 10.1007/s12021013-9213-2

Donohue, D. E., and Ascoli, G. A. (2008). A comparative computer simulation of dendritic morphology. PLoS Comput. Biol. 4:e1000089. doi: 10.1371/journal.pcbi.1000089

Donohue, D. E., and Ascoli, G. A. (2011). Automated reconstruction of neuronal morphology: an overview. Brain Res Rev. 67, 94-102. doi: 10.1016/j.brainresrev.2010.11.003

Dunn, J. C. (1973). A fuzzy relative of the ISODATA process and its use in detecting compact well-separated clusters. J. Cybern. 3, 32-57. doi: 10.1080/01969727308546046

Evans, R. C., and Polavaram, S. (2013). Growing a garden of neurons. Front. Neuroinform. 7:17. doi: 10.3389/fninf.2013.00017

Farley, C., and Raftery, A. E. (2006). MCLUST Version 3: an R Package for Normal Mixture Modeling and Model-Based Clustering. Seattle, WA: Department of Statistics, University of Washington. 
Goldberg, J. H., Tamas, G., Aronov, D., and Yuste, R. (2003). Calcium microdomains in aspiny dendrites. Neuron 40, 807-821. doi: 10.1016/S08966273(03)00714-1

Halavi, M., Hamilton, K. A., Parekh, R., and Ascoli, G. A. (2012). Digital reconstructions of neuronal morphology: three decades of research trends. Front. Neurosci. 6:49. doi: 10.3389/fnins.2012.00049

Kajiwara, R., Wouterlood, F. G., Sah, A., Boekel, A. J., Baks-te Bulte, L. T. G., and Witter, M. P. (2008). Convergence of entorhinal and CA3 inputs onto pyramidal neurons and interneurons in hippocampal area CA1-an anatomical study in the rat. Hippocampus 18, 266-280. doi: 10.1002/hipo.20385

Koene, R. A, Tijms, B., van Hees, P., Postma, F., de Ridder, A., Ramakers, G. J. A., et al. (2009). NETMORPH: a framework for the stochastic generation of large scale neuronal networks with realistic neuron morphologies. Neuroinformatics 7, 195-210. doi: 10.1007/s12021-009-9052-3

Koizumi, H., Koshiya, N., Chia, J. X., Cao, F., Nugent, J., Zhang, R., et al. (2013). Structural-functional properties of identified excitatory and inhibitory interneurons within pre-Botzinger complex respiratory microcircuits. J. Neurosci. 33, 2994-3009. doi: 10.1523/JNEUROSCI.4427-12.2013

Komendantov, A. K., and Ascoli, G. A. (2009). Dendritic excitability and neuronal morphology as determinants of synaptic efficacy. J. Neurophysiol. 101, 1847-1866. doi: 10.1152/jn.01235.2007

Kong, J.-H., Fish, D. R., Rockhill, R. L., and Masland, R. H. (2005). Diversity of ganglion cells in the mouse retina: unsupervised morphological classification and its limits. J. Comp. Neurol. 489, 293-310. doi: 10.1002/cne.20631

Lee, S., and Stevens, C. F. (2007). General design principle for scalable neural circuits in a vertebrate retina. Proc. Natl. Acad. Sci. U.S.A. 104, 12931-12935. doi: 10.1073/pnas.0705469104

London, M., and Häusser, M. (2005). Dendritic computation. Annu. Rev. Neurosci. 28, 503-532. doi: 10.1146/annurev.neuro.28.061604.135703

MacLean, J. N., Watson, B. O., Aaron, G. B., and Yuste, R. (2005). Internal dynamics determine the cortical response to thalamic stimulation. Neuron $48,811-823$. doi: 10.1016/j.neuron.2005.09.035

McGarry, L. M., Packer, A. M., Fino, E., Nikolenko, V., Sippy, T., and Yuste, R. (2010). Quantitative classification of somatostatin-positive neocortical interneurons identifies three interneuron subtypes. Front. Neural Circuits 4:12. doi: $10.3389 /$ fncir.2010.00012

Memelli, H., Torben-Nielsen, B., and Kozloski, J. (2013). Self-referential forces are sufficient to explain different dendritic morphologies. Front. Neuroinform. 7:1. doi: 10.3389/fninf.2013.00001

Nikolenko, V., Poskanzer, K. E., and Yuste, R. (2007). Two-photon photostimulation and imaging of neural circuits. Nat. Methods 4, 943-950. doi: 10.1038/nmeth1105

Oberlaender, M., de Kock, C. P., Bruno, R. M., Ramirez, A., Meyer, H. S.,Dercksen, V. J., et al. (2012). Cell type-specific three-dimensional structure of thalamocortical circuits in a column of rat vibrissal cortex. Cereb Cortex 22, 2375-2391. doi: 10.1093/cercor/bhr317

Parekh, R., and Ascoli, G. A. (2013). Neuronal morphology goes digital: a research hub for cellular and system neuroscience. Neuron 77, 1017-1038. doi: 10.1016/j.neuron.2013.03.008

Pyapali, G. K., Sik, A., Penttonen, M., Buzsaki, G., and Turner, D. A. (1998). Dendritic properties of hippocampal CA1 pyramidal neurons in the rat: intracellular staining in vivo and in vitro. J Comp Neurol. 391, 335-352.

Rocchi, M. B. L., Sisti, D., Albertini, M. C., and Teodori, L. (2007). Current trends in shape and texture analysis in neurology: aspects of the morphological substrate of volume and wiring transmission. Brain Res. Rev. 55, 97-107. doi: 10.1016/j.brainresrev.2007.04.001

Ropireddy, D., and Ascoli, G. A. (2011). Potential synaptic connectivity of different neurons onto pyramidal cells in a $3 \mathrm{D}$ reconstruction of the rat hippocampus. Front. Neuroinform. 5:5. doi: 10.3389/fninf.2011.00005

Santana, R., McGarry, L. M., Bielza, C., Larrañaga, P., and Yuste, R. (2013). Classification of neocortical interneurons using affinity propagation. Front. Neural Circuits 7:185. doi: 10.3389/fncir.2013.00185

Schneider, C. J., Bezaire, M., and Soltesz, I. (2012). Toward a full-scale computational model of the rat dentate gyrus. Front. Neural Circuits 6:83. doi 10.3389/fncir.2012.00083

Scorcioni, R., Polavaram, S., and Ascoli, G. A. (2008). L-Measure: a web-accessible tool for the analysis, comparison and search of digital reconstructions of neuronal morphologies. Nat. Protoc. 3, 866-876. doi: 10.1038/nprot.2008.51

Scorcioni, R., Lazarewicz, M. T., and Ascoli, G. A. (2004). Quantitative morphometry of hippocampal pyramidal cells: differences between anatomical classes and reconstructing laboratories. J. Comp. Neurol. 473, 177-193. doi: 10.1002/cne.20067

Shepherd, G. M. G., and Svoboda, K. (2005). Laminar and columnar organization of ascending excitatory projections to layer $2 / 3$ pyramidal neurons in rat barrel cortex. J. Neurosci. 25, 5670-5679. doi: 10.1523/JNEUROSCI.117305.2005

Smit-Rigter, L. A., Noorlander, C. W., von Oerthel, L., Chameau, P., Smidt, M. P., and van Hooft, J. A. (2012). Prenatal fluoxetine exposure induces life-long serotonin 5-HT3 receptor-dependent cortical abnormalities and anxiety-like behaviour. Neuropharmacology 62, 865-870. doi: 10.1016/j.neuropharm.2011.09.015

Snider, J., Pillai, A., and Stevens, C. F. (2010). A universal property of axonal and dendritic arbors. Neuron 66, 45-56. doi: 10.1016/j.neuron.2010.02.013

Soloway, A. S., Pucak, M. L., Melchitzky, D. S., and Lewis, D. A. (2002). Dendritic morphology of callosal and ipsilateral projection neurons in monkey prefrontal cortex. Neuroscience 109, 461-471. doi: 10.1016/S0306-4522(01) 00507-3

Teeter, C. M., and Stevens, C. F. (2011). A general principle of neural arbor branch density. Curr. Biol. 21, 2105-2108. doi: 10.1016/j.cub.2011.11.013

Ting, C.-Y., McQueen, P. G., Pandya, N., Lin, T.-Y., Yang, M., Reddy, O. V., et al. (2014). Photoreceptor-derived activin promotes dendritic termination and restricts the receptive fields of first-order interneurons in Drosophila. Neuron 81, 830-846. doi: 10.1016/j.neuron.2013.12.012

Thomson, A. M., and Armstrong, W. E. (2011). Biocytin-labelling and its impact on late 20th century studies of cortical circuitry. Brain Res Rev. 66, 43-53. doi: 10.1016/j.brainresrev.2010.04.004

Uylings, H. B. M., and van Pelt, J. (2002). Measures for quantifying dendritic arborizations. Network 13, 397-414. doi: 10.1088/0954-898X/13/3/309

Van Ooyen, A. (2011). Using theoretical models to analyse neural development. Nat. Rev. Neurosci. 12, 311-326. doi: 10.1038/nrn3031

Van Ooyen, A., Duijnhouwer, J., Remme, M. W. H., and van Pelt, J. (2002). The effect of dendritic topology on firing patterns in model neurons. Network 13 , 311-325. doi: 10.1088/0954-898X/13/3/304

Van Pelt, J., Dityatev, A. E., and Uylings, H. B. (1997). Natural variability in the number of dendritic segments: model-based inferences about branching during neurite outgrowth. J. Comp. Neurol. 387, 325-340.

Weiler, N., Wood, L., Yu, J., Solla, S. A., and Shepherd, G. M. G. (2008). Top-down laminar organization of the excitatory network in motor cortex. Nat. Neurosci. 11, 360-366. doi: 10.1038/nn2049

Wen, Q., and Chklovskii, D. B. (2008). A cost-benefit analysis of neuronal morphology. J. Neurophysiol. 99, 2320-2328. doi: 10.1152/jn.00280.2007

Woodruff, A., Xu, Q., Anderson, S., and Yuste, R. (2009). Depolarizing effect of neocortical chandelier neurons. Front. Neural Circuits 3:15. doi: 10.3389/neuro.04.015.2009

Wright, S. N., Kochunov, P., Mut, F., Bergamino, M., Brown, K. M., Mazziotta, J. C., et al. (2013). Digital reconstruction and morphometric analysis of human brain arterial vasculature from magnetic resonance angiography. Neuroimage 82, 170-181. doi: 10.1016/j.neuroimage.2013.05.089

Yates, D. S., Moore, D. S., and McCabe, G. P. (1999). The Practice of Statistics: TI-83 Graphing Calculator Enhanced. New York, NY: W.H. Freeman.

Zawadzki, K., Feenders, C., Viana, M. P., Kaiser, M., and Costa Lda, F. (2012). Morphological homogeneity of neurons: searching for outlier neuronal cells. Neuroinformatics 10, 379-389. doi: 10.1007/s12021-012-9150-5

Conflict of Interest Statement: The authors declare that the research was conducted in the absence of any commercial or financial relationships that could be construed as a potential conflict of interest.

Received: 08 June 2014; accepted: 06 November 2014; published online: 04 December 2014.

Citation: Polavaram S, Gillette TA, Parekh R and Ascoli GA (2014) Statistical anal$y$ sis and data mining of digital reconstructions of dendritic morphologies. Front. Neuroanat. 8:138. doi: 10.3389/fnana.2014.00138

This article was submitted to the journal Frontiers in Neuroanatomy.

Copyright (C) 2014 Polavaram, Gillette, Parekh and Ascoli. This is an open-access article distributed under the terms of the Creative Commons Attribution License (CC BY). The use, distribution or reproduction in other forums is permitted, provided the original author(s) or licensor are credited and that the original publication in this journal is cited, in accordance with accepted academic practice. No use, distribution or reproduction is permitted which does not comply with these terms. 